\title{
WPVS007: evidence for accelerating wind in a narrow line Seyfert 1 galaxy?
}

\section{E. M. Cooper ${ }^{*}{ }^{a}$ K. M. Leighly, ${ }^{a}$ F. Hamann ${ }^{b}$ and D. Grupe ${ }^{c}$}

${ }^{a}$ Homer L. Dodge Department of Physics and Astronomy, The University of Oklahoma, USA

${ }^{b}$ Department of Astronomy, University of Florida, USA

${ }^{c}$ Department of Astronomy and Astrophysics, Pennsylvania State University, USA

Email: cooperenhn.ou.edu

\begin{abstract}
Outflows play a significant role in the physics of active galaxies by shaping galactic evolution, enriching and mixing the intergalactic medium, and potentially removing angular momentum. WPVS007 is a low-luminosity, Narrow Line Seyfert 1 (NLS1) galaxy with a remarkable history of an emerging and evolving outflow. In a 1996 Hubble Space Telescope (HST) observation, WPVS007 had miniBALs with $v_{\max } \sim 1000 \mathrm{~km} \mathrm{~s}^{-1}$. By the 2003 FUSE observation [1], WPVS007 developed a BAL outflow with $v_{\max } \sim 6000 \mathrm{~km} \mathrm{~s}^{-1}$. We present the 2010 HST COS spectrum, which revealed that maximum velocity of the outflow in WPVS007 had increased substantially, to $v_{\max } \geq 10,000 \mathrm{~km} \mathrm{~s}^{-1}$. The higher maximum velocity may indicate acceleration of the outflowing gas. Such a change in the maximum velocity is unprecedented, since BAL variability is typically confined to differences in apparent optical depth. High velocity outflows are unexpected in low-luminosity active galaxies, like WPVS007, making the observed outflow extraordinary.
\end{abstract}

Nuclei of Seyfert galaxies and QSOs - Central engine \& conditions of star formation November 6-8, 2012

Max-Planck-Insitut für Radioastronomie (MPIfR), Bonn, Germany

* Speaker. 


\section{Introduction}

WPVS007 is a low-luminosity Narrow-line Seyfert $1\left(\mathrm{z}=0.02882, \mathrm{M}_{\mathrm{V}}=-18.8\right)$ galaxy with an exceptional history. When HST observed WPVS007 in the UV in 1996, the spectrum showed strong, narrow emission lines similar to other low-luminosity NLS1s and low-velocity broad absorption lines (miniBALs) in UV resonance lines ( $\mathrm{N}$ V and $\mathrm{C} \mathrm{IV}$, among others), but no true broad absorption lines (BALs). By the 2003 FUSE observation [1], WVPS007 developed a broad absorption line outflow with a $v_{\max } \sim 6,000 \mathrm{~km} \mathrm{~s}^{-1}$. We present new HST observations that indicate the BALs in WPVS007 have evolved further, extending to higher velocities.

\section{Data}

We present new WPVS007 data from the Hubble Space Telescope Cosmic Origins Spectrograph. The observation was taken June 11, 2010. The instrument was configured with the FUV detector, PSA aperture and G140L grating at a central wavelength of $1230 \AA$. Three exposures were taken in each of three nod positions for a total exposure time of 5060.576s.
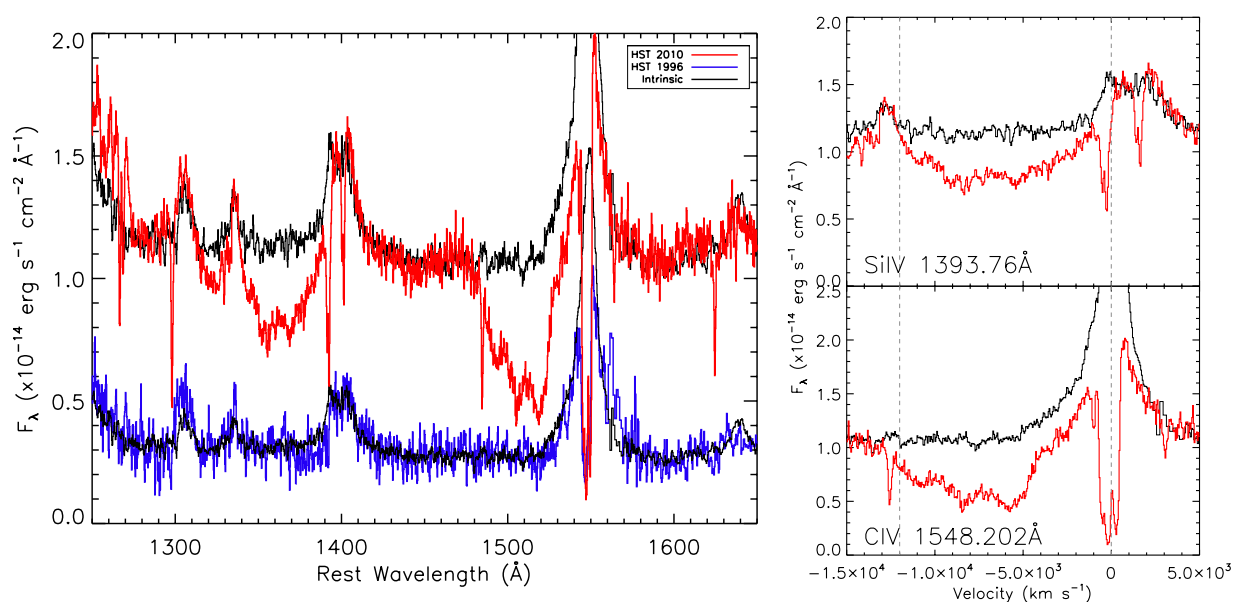

Figure 1: HST COS 2010 WPVS 007 spectra. (Left) (red) 2010 HST COS, (blue) 1996 HST FOS, (black) scaled mean Mrk 335 \& Mrk 439 template from [1]. (Right) Absorption profiles in velocity space for Si IV \& C IV. $0 \mathrm{~km} \mathrm{~s}^{-1}$ represents the blue line in each doublet. $12,000 \mathrm{~km} \mathrm{~s}^{-1}$ is also plotted for reference.

The COS FUV G140L data are currently not flat calibrated by the pipeline. Monitoring observations of the white dwarf WD0947+857 indicate flat variations are small (within the noise) for segment A, but contain significant structure in segment B. We are currently working on a 1D flat to correct for these variations. We apply a wavelength and new sensitivity curve to segment B as indicated by private communication from the STScI help center to further address the strongly wavelength dependent throughput. The segment A data are plotted on the left in Figure 1. The Si IV and C IV BALs appear clearly and unambiguously, each with a $v_{\max } \geq 12,000 \mathrm{~km} \mathrm{~s}^{-1}$. Velocity profiles are plotted on the right in Figure 1, with $0 \mathrm{~km} \mathrm{~s}^{-1}$ indicating the blue line of the doublet in each plot.

The P v BAL gives us the only direct comparison with the 2003 FUSE spectrum. While comparison with the 2003 FUSE spectrum clearly shows the the P v BAL shifting to shorter wavelengths (higher velocities), continuum placement plays a significant role in the measurement of 
the maximum and minimum velocities. Using the same placement of the HST FOS composite scaled to the FUSE data as in [1], conservative estimates for $\mathrm{P} \mathrm{V}$ are $v_{\max } \sim 8,000 \mathrm{~km} \mathrm{~s}^{-1}$ and $v_{\text {min }} \sim 2,000 \mathrm{~km} \mathrm{~s}^{-1}$, as compared with $\sim 6,000 \mathrm{~km} \mathrm{~s}^{-1}$ and $\sim 200 \mathrm{~km} \mathrm{~s}^{-1}$ for the FUSE observation.

A preliminary extraction of the $\mathrm{P} v$ optical depth profile indicates that if the FUSE P V optical depth profile is shifted to higher velocities and scaled down, it resembles the new optical depth profile, with some obvious deviations. The similarity in profiles suggests a possible acceleration of the outflow to higher velocities rather than a change in the trough depth owing to changes in ionization or covering factor.

\section{Constraints on the Absorption Region Size}

That we see $\mathrm{P} \mathrm{V}$ and not $\mathrm{He} \mathrm{I}^{*}$ in WPVS007 (Grupe et al., in preparation) means we can put constraints on the size of the absorbing region. Using the sum-ofblackbodies accretion disk model, we estimate the size of the $1120 \AA$ continuum emitting region to be $3.0 \times 10^{-5} \mathrm{pc}, \sim 21 \mathrm{x}$ smaller than that for $10830 \AA\left(6.1 \times 10^{-4} \mathrm{pc}\right)$. In comparison, the BLR radius is estimated to be $8.9 \times 10^{-4} \mathrm{pc}$ for this $4.1 \times 10^{6}$ solar mass black hole, radiating at $9.6 \%$ of the Eddington luminosity [1]. From Figure 2, we know that wherever we see $\mathrm{P} \mathrm{V}$ we must also see He I*. This means the absorption region does not cover the $10830 \AA$ continuum emit-

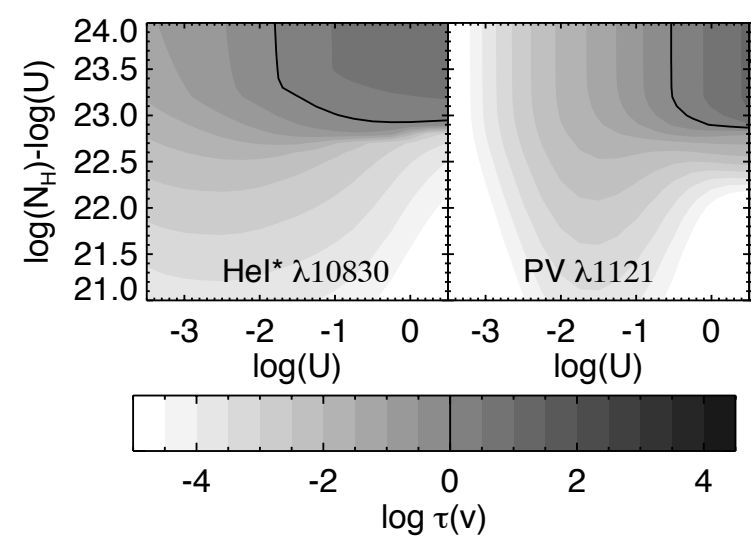

Figure 2: Optical depth contours for log column density vs. log ionization parameter for a line with a velocity width of $10,000 \mathrm{~km} \mathrm{~s}^{-1}$. The contours show that everywhere we see $\mathrm{P}$ V, we must also see He I*. ting region. Either the outflow is located at a smaller radius than that of the $10830 \AA$ continuum emitting region or it is a narrow bullet only along our line of sight. If it is the latter, then the global covering fraction would have to be much lower in WPVS007 than the usually estimated value of 0.2 based on the fraction of quasars with BALs.

\section{Future Work}

The approved HST Cycle 20 follow-up observations of WPVS007 will help us determine whether the extension of the BAL outflow to higher velocities is caused by genuine acceleration or a change in optical depth, covering fraction or ionization. We proposed two follow-up observations, spaced six months apart so that if the shape of the outflow is preserved, but shifted to higher velocities, we will have seen acceleration of outflowing gas in WPVS007.

\section{References}

[1] K.M. Leighly et al., Emergence of a broad absorption line outflow in the narrow-line Seyfert 1 Galaxy WPVS 007, ApJ 701 (2009) 176 [astro-ph.CO/0906.2149] 